\title{
Analysis of MreB interactors in Chlamydia reveals a RodZ homolog but fails to detect an interaction with MraY
}

\author{
Scot P. Ouellette ${ }^{1,2 *}$, Kelsey J. Rueden ${ }^{2}$, Emilie Gauliard ${ }^{1,3}$, Logan Persons ${ }^{4}$, Piet A. de Boer ${ }^{4}$ and \\ Daniel Ladant ${ }^{1}$ \\ 1 Unité de Biochimie des Interactions Macromoléculaires, Département de Biologie Structurale et Chimie, Institut Pasteur, CNRS UMR 3528, Paris, France \\ 2 Division of Basic Biomedical Sciences, Sanford School of Medicine, University of South Dakota, Vermillion, SD, USA \\ ${ }^{3}$ Université Paris Diderot, Sorbonne Paris Cité, Cellule Pasteur, Paris, France \\ ${ }^{4}$ Department of Molecular Biology and Microbiology, School of Medicine, Case Western Reserve University, Cleveland, OH, USA
}

\section{Edited by:}

Biswarup Mukhopadhyay, Virginia

Tech, USA

Reviewed by:

Hans-Georg Koch, Albert-Ludwigs University Freiburg, Germany

Dan Rockey, Oregon State

University, USA

*Correspondence:

Scot P. Ouellette, Division of Basic Biomedical Sciences, Sanford

School of Medicine, University of South Dakota, 414 E. Clark St.

LM359, Vermillion, SD 57069, USA e-mail: scot.ouellette@usd.edu
Chlamydia is an obligate intracellular bacterial pathogen that has significantly reduced its genome in adapting to the intracellular environment. One class of genes for which the bacterium has few annotated examples is cell division, and Chlamydia lacks FtsZ, a central coordinator of the division apparatus. We have previously implicated MreB as a potential substitute for FtsZ in Chlamydia (Ouellette et al., 2012). Thus, to identify new chlamydial cell division components, we searched for proteins that interacted with MreB. We performed a small-scale screen using a Gateway ${ }^{\circledR}$ compatible version of the Bacterial

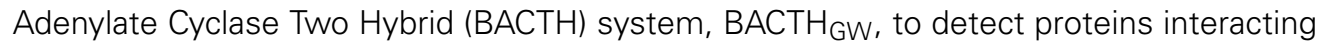
with chlamydial MreB and identified a RodZ (YfgA) homolog. The chlamydial RodZ aligns well with the cytoplasmic domain of $E$. coli RodZ but lacks the periplasmic domain that is dispensable for rod cell shape maintenance in $E$. coli. The expression pattern of $y f g A / r o d Z$ was similar to that of $m r e B$ and $f t s l$, suggesting that these genes may operate in a common functional pathway. The chlamydial RodZ correctly localized to the membrane of $E$. coli but was unable to complement an E. coli rodZ mutant strain, likely because of the inability of chlamydial RodZ to interact with the native E. coli MreB. Finally, we also tested whether chlamydial MreB could interact with MraY, as suggested by Gaballah et al. (2011). However, we did not detect an interaction between these proteins even when using an implementation of the BACTH system to allow native orientation of the $\mathrm{N}$ - and C-termini of MraY in the periplasm. Thus, further work will be needed to establish this proposed interaction. In sum, we have added to the repertoire of potential cell division proteins of Chlamydia.

Keywords: Chlamydia, bacterial two-hybrid system, protein-protein interactions, cell division, RodZ, MreB

\section{INTRODUCTION}

Chlamydia trachomatis is an obligate intracellular bacterial pathogen that causes blinding trachoma and sexually transmitted diseases (Schachter et al., 1973; Mabey et al., 2003). Chlamydia has a unique developmental cycle that alternates between an extracellular, infectious form (the elementary body or EB that mediates attachment to and uptake by susceptible host cells) and an intracellular, non-infectious form (the reticulate body or $\mathrm{RB}$ that grows and divides by binary fission) (see AbdelRahman and Belland, 2005 for review). A feature of Chlamydia intracellular growth is that it remains within a pathogen-specified vacuole, called an inclusion, for the duration of its developmental cycle. The inclusion remains non-fusogenic with the endolysosomal system but is highly interactive with host cell trafficking pathways.

One unusual aspect of chlamydial microbiology is the lack of a homolog for the essential cell division protein, FtsZ, a tubulin-like homolog (Mukherjee et al., 1993). However, chlamydiae do encode homologs for rod-shape determining proteins in spite of their coccoid shape (Ouellette et al., 2012). We recently hypothesized that MreB, an actin-like homolog critical in the establishment and maintenance of the rod shape of bacilli (Doi et al., 1988; Bork et al., 1992), may substitute for FtsZ in Chlamydia and presented evidence that chlamydial MreB is necessary for division of this bacterium (Ouellette et al., 2012).

MreB interacts with a number of proteins, one of which is YfgA. YfgA has been characterized as a rod-shape determining protein called RodZ (Shiomi et al., 2008; Alyahya et al., 2009; Bendezú et al., 2009). E. coli YfgA/RodZ contains a cytoplasmic region, which encodes a helix-turn-helix (HTH) domain that is important for interactions with $\mathrm{MreB}$, a transmembrane domain, and a periplasmic domain (Bendezú et al., 2009; van den Ent et al., 2010). Very recently, a RodZ homolog in Waddlia (an organism related to the Chlamydiales), was implicated in division of that organism by recruiting MreB to the division plane (Jacquier et al., 2014). The authors suggested that peptidoglycan precursors are recruited to the division plane prior to MreB in part because MreB is purported to interact with components of the peptidoglycan enzymatic components including MraY (Gaballah et al., 2011). 
As chlamydial MreB may serve an important function in the division of this bacterium, we sought to identify potential interaction partners by using the Bacterial Adenylate Cyclase Two Hybrid (BACTH) system as a surrogate approach to study molecular details of Chlamydia biology. Indeed, owing to the unusual and complex growth requirements of Chlamydia, there are currently no molecular tools for generating conditional depletion systems in this pathogen, and analysis of protein-protein interactions in vivo is problematic owing to the large amount of background host protein. The BACTH system relies on the reconstitution of adenylate cyclase activity in $E$. coli by fusing proteins of interest to two complementary fragments, T25, and T18, from the catalytic domain of the adenylate cyclase toxin of Bordetella pertussis (Karimova et al., 1998). The fragments are inactive when co-expressed separately, but when they are fused to proteins that interact, then the T25 and T18 fragments are brought into close proximity to allow functional complementation of enzyme activity. As the BACTH system relies on the generation of a diffusible regulatory molecule (i.e., cyclic AMP from the reconstituted cyclase activity), it allows for the separation of the protein-protein interaction and the transcriptional apparatus. The system is thus appropriate to study a wide variety of protein-protein interactions, in particular those occurring between integral membrane proteins. This technique has been extensively used to characterize protein-protein interactions in vivo within a variety of contexts (see Battesti and Bouveret, 2012 for review), and we recently described a Gateway ${ }^{\circledR}$ compatible version of this system $\left(\mathrm{BACTH}_{\mathrm{GW}}\right)$ that facilitates analysis of targeted screening assays (Ouellette et al., 2014).

Here, we identified a chlamydial homolog of RodZ/YfgA, Ct009, by performing a small-scale screen from a library of Gateway ${ }^{\circledR}$ clones to look for interaction partners of chlamydial MreB. We further demonstrated the specificity of the chlamydial MreB/RodZ association by mutating two conserved aromatic residues of RodZ and showing that this abolished their interaction. Chlamydial rod $Z$ is expressed as an RB-specific gene. Chlamydial RodZ failed to complement an E. coli rodZ mutant likely due to decreased or defective interactions with $E$. coli MreB. We also tested the interaction of chlamydial MreB with the chlamydial homolog of MraY, an integral membrane protein that catalyzes the synthesis of the first lipid intermediate (Lipid I) of the bacterial cell wall peptidoglycan. However, at variance with a prior report that also used the BACTH assay (Gaballah et al., 2011), we found that MreB did not interact with MraY. Therefore, the association of the putative chlamydial division components with the peptidoglycan synthesis machinery remains to be conclusively established. In sum, a chlamydial RodZ homolog adds to the repertoire of rod-shape determining proteins in this coccoid bacterium.

\section{MATERIALS AND METHODS CLONING}

A list of primers and plasmids used in this study can be viewed in Supplemental Table S1. Standard protocols were used for PCR, digestion, ligation, transformation, and plasmid preparation. E. coli MG1655 or C. trachomatis L2 genomic DNA was used as template for PCR when necessary. PCR was performed using the high-fidelity Phusion DNA polymerase (Thermo Fisher, Illkirch, France), purified with a PCR purification kit (Qiagen, Courtaboeuf, France), and, when necessary, digested with the indicated restriction enzymes (FastDigest; Thermo Fisher). Empty vectors were digested with the indicated restriction enzymes in the presence of alkaline phosphatase (FastAP; Thermo Fisher). Ligation reactions were performed using T4 DNA ligase (Thermo Fisher). Transformations were performed in chemically competent E. coli XL1-Blue (Stratagene [Agilent], Santa Clara, CA) cells and plated on selective antibiotics in LB agar in the presence of $0.4 \%$ glucose to repress expression. The Q5 site-directed mutagenesis kit was used, with the indicated mutagenesis primers in Supplemental Table S1, to mutate the MreB interaction residues in $\operatorname{rod} Z$ following the manufacturer's guidelines (New England Biolabs, Ipswich, MA). Medium and other chemicals were obtained from Sigma (St. Louis, MO) except where noted.

\section{GATEWAY ${ }^{\circledR}$ RECOMBINATION REACTIONS}

The BP and LR (refers to the att sites) recombination reactions were performed according to the manufacturer's guidelines (Invitrogen [Life Technologies], Grand Island, NY). For all chlamydial full-length constructs except $m r e B$, the indicated gene was obtained from a partial library consisting of 280 C. trachomatis serovar D ORFs cloned into the pDONR221 vector as constructed and sequence-verified by the Pathogen Functional Genomic Resource Center (http://pfgrc.jcvi.org). For chlamydial $m r e B$, an $a t t B$-flanked PCR product was used in a BP reaction to insert the gene of interest into the attP-flanked pDONR221 to generate an $a t t L$-flanked ORF, which was then sequence-verified. The attL-flanked ORF was then recombined into the $a t t R$-flanked BACTH-DEST plasmids using the LR reaction to generate an attB-flanked ORF within the BACTH vectors (Ouellette et al., 2014). The insert was subsequently sequenced for orientation.

\section{BACTH AND $\beta$-GALACTOSIDASE ASSAYS}

BACTH interactions were performed as previously described (Karimova et al., 2005) using the adenylate cyclase mutant $(\Delta c y a)$ strain of E. coli, DHT1. Briefly, chemically competent DHT1 were co-transformed with each plasmid to be tested and plated on M63 minimal medium agar containing selective antibiotics, $40 \mu \mathrm{g} / \mathrm{mL}$ X-gal (Thermo Fisher), $0.5 \mathrm{mM}$ IPTG (Thermo Fisher), 0.04\% casamino acids, and 0.2\% maltose (Karimova et al., 1998). Plates were incubated at $30^{\circ} \mathrm{C}$ for up to 5 days for interactions. Only bacteria exhibiting adenylate cyclase activity are able to support robust growth on minimal medium with maltose as the sole sugar source. A positive control of the cytosolic zip constructs (Karimova et al., 1998) was used whereas negative controls included tests vs. either the empty BACTH plasmids or non-related chlamydial inner membrane proteins, Ct471 or GlnP/Ct129 (Ouellette et al., 2012). Eight colonies from each plate were cultured for $24 \mathrm{~h}$ at $30^{\circ} \mathrm{C}$ in 96 -well plate format in $300 \mu \mathrm{L}$ minimal medium broth containing selective antibiotics, IPTG, casamino acids, and maltose. These cultures were diluted to $1 \mathrm{~mL}$ in minimal medium (without supplements) the following day. A blank of medium only was also included for background levels. $200 \mu \mathrm{L}$ was used for $\mathrm{OD}_{600}$ measurement and $200 \mu \mathrm{L}$ was used to 
lyse the bacteria, using chloroform and sodium dodecyl sulfate, for the $\beta$-galactosidase measurement and subsequently incubated with $0.4 \%$ ONPG for $10-20 \mathrm{~min}$. The reaction was stopped by adding $\mathrm{Na}_{2} \mathrm{CO}_{3}$ and visualized at $\mathrm{OD}_{405} \cdot \beta$-galactosidase activity is expressed as $1000^{*}\left(\left[\mathrm{OD}_{405}-\right.\right.$ blank $\left.] /\left[\mathrm{OD}_{600}-\mathrm{blank}\right]\right) / \mathrm{min}$. All interaction tests were performed a minimum of two times on plates with eight colonies from each plate analyzed for $\beta$-galactosidase activity.

\section{RT-qPCR}

Assays to quantify the indicated transcripts were performed essentially as described previously (Ouellette et al., 2005, 2006). Briefly, total RNA was collected from infected cells at the indicated times using Trizol (Invitrogen) and treated with Turbo DNAfree (Ambion [Life Technologies]) to remove contaminating DNA, according to the manufacturer's guidelines. $1 \mu \mathrm{g}$ DNA-free RNA was reverse-transcribed with random nonamers (New England Biolabs, Ipswich, MA) using SuperScript III RT (Invitrogen) according to the manufacturer's instructions. Equal volumes of cDNA were used in qPCR reactions with SYBR Green (Quanta Biosciences, Gaithersburg, MD) and measured on an ABI 7300 system (Applied Biosystems [Life Technologies]). Duplicate DNA samples were collected from the same experiment using DNeasy Tissue kit (Qiagen). Chlamydial genomes were quantified from equal amounts of total DNA by qPCR as above and used to normalize transcript data as described (Ouellette et al., 2005, 2006).

\section{E. COLI RodZ MUTANT AND COMPLEMENTATION}

The $\operatorname{rod} Z$ mutant strain (FB60/pTB63) has been previously characterized (Bendezú et al., 2009). This strain or DH5 $\alpha$ were transformed with plasmids pLP173 (expressing GFP-CtrRodZ), pLP174 (expressing GFP-Ec-RodZ), or the empty vector pMLB1113 $\mathrm{H} 3$ (bla lacI ${ }^{q} \mathrm{P}_{\text {lac }}:$ :-). Strains were grown overnight at $30^{\circ} \mathrm{C}$ in $\mathrm{LB}$ medium with $50 \mu \mathrm{g} / \mathrm{mL}$ ampicillin and $0.2 \%$ glucose (and $5 \mu \mathrm{g} / \mathrm{mL}$ tetracycline for strains harboring pTB63). Overnight cultures were diluted 1:100 in M9-maltose medium with $50 \mu \mathrm{g} / \mathrm{mL}$ ampicillin and $25 \mu \mathrm{M}$ IPTG and grown at $30^{\circ} \mathrm{C}$ until mid-log phase $\left(\mathrm{OD}_{600}=0.4-0.6\right)$. Samples were visualized on a Zeiss Axioplan-2 microscope and imaged at $1000 \times$ magnification as described (Johnson et al., 2002).

\section{RESULTS}

\section{INTERACTIONS OF THE BACTERIAL CYTOSKELETAL PROTEIN, MreB}

MreB is a bacterial homolog of actin and is thought to serve an essential role in maintaining the rod-shape of bacillus bacteria. MreB can form filaments and use ATP, like actin (van den Ent et al., 2001). To characterize the interacting properties of chlamydial MreB with the BACTH system, we cloned the full-length $m r e B$ either into the Gateway ${ }^{\circledR}$ compatible $\left(\mathrm{BACTH}_{\mathrm{GW}}\right)$ vectors or into the original BACTH vectors (although we could not obtain a correct insertion of MreB into the high-copy pUT18C vector). Two-hybrid assays revealed that each of the chlamydial MreB T25 clones, expressed from $p K T 25-m r e B$ or $p S T 25-m r e B(G W)$, was capable of interacting with the T18 MreB Gateway ${ }^{\circledR}$ clone, expressed from $p U T 18 C$-mreB $(G W)$ (Figure 1), thus confirming the ability of chlamydial MreB to oligomerize.

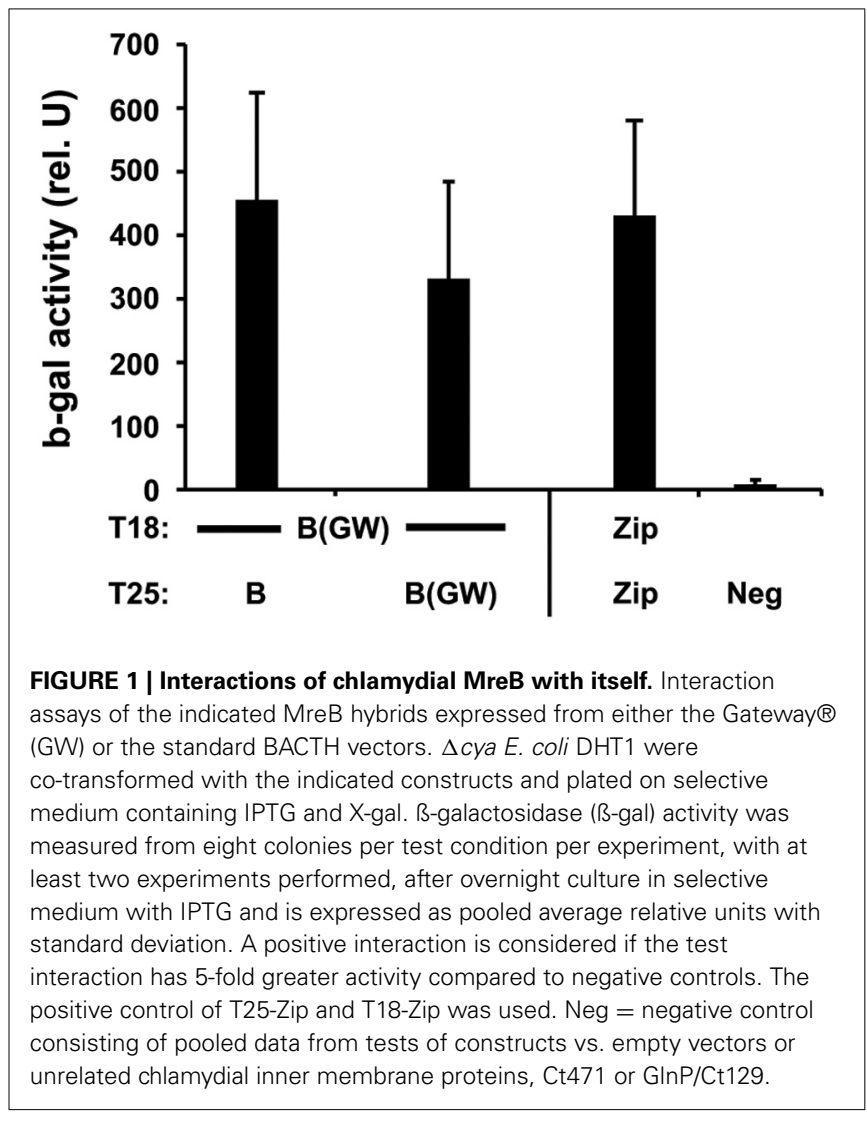

Given the proposed role of chlamydial MreB as a functional substitute for FtsZ (Ouellette et al., 2012) and the paucity of annotated cell division proteins in this bacterium, we wanted to identify interaction partners of MreB since such proteins might function in cell division. To this end, we used our Gateway ${ }^{\circledR}$ library of chlamydial ORFs to perform a small-scale biased screen using chlamydial MreB as bait. Table 1 summarizes the results. Amongst the 21 genes we analyzed, we identified only three positive interactions, two of which were previously described and included as positive controls (Ouellette et al., 2012): MreB (Ct709), FtsK (Ct739), and Ct009. However, we did not detect an interaction with MraY or MurG, at variance with a previous report from Gaballah et al. (2011).

To verify the predicted topology of chlamydial MraY, we took advantage of recent work showing that the charge characteristics at the C-terminus of the last transmembrane (TM) domain of a polytopic membrane protein determine its orientation in the membrane (Seppala et al., 2010). We thus inserted the C-terminal 26 amino acids encoding the last TM domain (TM10) of chlamydial MraY, as predicted by TOPCONS (Supplemental Figure S1; Bernsel et al., 2009), between the T25 or T18 fragment and the leucine zipper (Zip) domain as described (Figure 2A; Ouellette et al., 2014). We then tested with the BACTH system whether these fusion proteins (T25-TM10-zip and T18-TM10-zip) interacted with either a cytosolic Zip (e.g., T25-Zip) or a periplasmic zip fused to the T25 domain via the first TM domain of $E$. coli OppB (e.g., T25-TM-zip; Ouellette et al., 2014). As shown in Figure 2B, the T25-TM10-zip interacted specifically with the 
Table 1 | Small-scale BACTH screen to identify proteins that interact with chlamydial MreB.

\begin{tabular}{|c|c|c|}
\hline Gene ID ${ }^{1}$ & Annotation & Interaction \\
\hline Ct005 & Hypothetical & - \\
\hline Ct009 & $y f g A$ & + \\
\hline Ct012 & $y b b P$ & - \\
\hline Ct129- & $g \ln P$ & - \\
\hline Ct144 & Hypothetical & - \\
\hline Ct174 & Hypothetical & - \\
\hline Ct270- & $\mathrm{ftsl} / \mathrm{pbp3}$ & - \\
\hline Ct277 & Hypothetical & - \\
\hline Ct303 & Hypothetical & - \\
\hline Ct357 & Hypothetical & - \\
\hline Ct446 & euo & - \\
\hline Ct471 & Hypothetical & - \\
\hline Ct482 & Hypothetical & - \\
\hline Ct605 & Hypothetical & - \\
\hline Ct682- & pbp2 & - \\
\hline Ct709+ & mreB & + \\
\hline Ct726 & $\operatorname{rod} A$ & - \\
\hline Ct739+ & ftsK & + \\
\hline Ct756 & murF & - \\
\hline Ct757\# & $\operatorname{mraY}$ & - \\
\hline Ct760 & $f t s W$ & - \\
\hline Ct761"\# & murG & - \\
\hline
\end{tabular}

${ }^{1}$ Using the numbering scheme of Stephens et al. (1998) at http://stdgen. northwestern.edu. + Included as positive or - negative control (Ouellette et al., 2012). "Reported as BACTH interacting partner by Gaballah et al. (2011).

T18-TM-zip but not T18-Zip, and, conversely, the T18-TM10-zip interacted strongly with the T25-TM-zip and to a much lower extent with the T25-Zip. This indicates that the chlamydial MraY TM10 segment is indeed driving the zip motif to the periplasm. Therefore, Chlamydia MraY encodes its C-terminus, and by extension, its $\mathrm{N}$-terminus, in the periplasm in accordance with the topology of E. coli MraY (Bouhss et al., 1999).

We next re-examined whether chlamydial MreB was capable of interacting with MraY using the $\mathrm{BACTH}_{\mathrm{GW}}$ system with (and without) the additional OppB TM to favor the native topology of MraY (Ouellette et al., 2014). The chlamydial mraY gene was recombined into all $\mathrm{BACTH}_{\mathrm{GW}}$ vectors: pST25-DEST, pUT18CDEST, pSNT25-DEST, pSTM25-DEST, and pUTM18C-DEST. We then transformed the $\Delta c y a E$. coli with different pair-wise combinations of the MreB and MraY constructs and assayed the $\beta$-galactosidase activity to quantify the interactions between these different hybrid proteins. We did not detect an interaction between MreB and MraY when MraY was expressed from any $\mathrm{BACTH}_{\mathrm{GW}}$ vector (Figure $2 \mathrm{C}$ and data not shown) and neither did we detect a specific interaction of E. coli MreB with its MraY (data not shown). Therefore, these BACTH analyses do not support an association between MreB and MraY.

\section{IDENTIFICATION OF A CHLAMYDIAL RodZ HOMOLOG}

We detected, and subsequently validated, from our initial screen an interaction with the chlamydial protein Ct009, annotated as YfgA (Stephens et al., 1998; Figure 3A). An alignment of Ct009 against E. coli RodZ showed that Ct009 aligns quite well with RodZ in spite of the low level of identity between the proteins (25\% identical/40\% similar over $43 \%$ of E. coli RodZ; $\mathrm{E}$ value $=6 \mathrm{e}-07 ;$ Figure 3B). Interestingly, Chlamydia RodZ lacks the large periplasmic domain that was shown to be dispensable for cell shape maintenance in E. coli (Bendezú et al., 2009). Ct009 homologs are present in all chlamydial genomes (Figure 3C and data not shown), an important consideration for a potential cell division component. Within the Chlamydiales, the Protochlamydia RodZ homolog grouped outside the other chlamydial species whereas the animal species clustered with each other and were separate from C. trachomatis (Figure 3C).

In E. coli, two aromatic residues (F60 and Y64) within the HTH domain have been shown to be critical for its interaction with MreB (van den Ent et al., 2010). To further validate the specific interaction of Ct009 with $\mathrm{MreB}$, we mutated the corresponding residues (Y66 and F70) within the HTH domain of Ct009 (indicated by + in Figure 3C). We then tested these variants (Y66A and F70A) against MreB in the BACTH assay. As shown in Figure 3D, we did not detect any interaction between these Ct009 variants and MreB, demonstrating the specificity of the Ct009/MreB association. In sum, these data strongly support the conclusion that Ct009 is a chlamydial RodZ homolog.

\section{ct009/rodZ IS TRANSCRIBED AS AN RB-SPECIFIC GENE}

As a first step to determining a putative function for Ct009/RodZ, we measured its transcription during the chlamydial developmental cycle to assign it a temporal pattern of expression. RNA was collected at various time points to reflect the differentiation of $\mathrm{EB}$ to $\mathrm{RB}$ (i.e., early cycle), RB growth and division (i.e., mid cycle), and re-differentiation from RB to $\mathrm{EB}$ (i.e., late cycle) (Shaw et al., 2000). A panel of representative genes of these temporal classes was examined as a control with euo representing early (Wichlan and Hatch, 1993), ftsI (a bona fide cell division gene) representing mid, and $o m c B$ representing late cycle stages (Everett and Hatch, 1991). As can be seen in Figure 4, ct009/rodZ transcription most closely resembles the mid cycle transcriptional pattern of ftsI and markedly differs from the expression pattern of genes specific to the $\mathrm{EB}$ to $\mathrm{RB}$ (euo) or RB to $\mathrm{EB}(\mathrm{omcB})$ transition. Furthermore, the pattern of $c t 009 / \mathrm{rod} Z$ expression closely resembled that of $m r e B$, its binding partner. Therefore, we conclude that, like MreB, Ct009/RodZ functions as an RB-specific product and most likely as a cell division-related protein.

\section{Ct009/RodZ LOCALIZES TO MEMBRANES IN E. COLI BUT FAILS TO COMPLEMENT AN E. COLI rodZ MUTANT}

Since division proteins are essential for viability, putative candidate division components are typically investigated using conditional depletion systems, temperature-sensitive mutants, or by over-expressing key proteins of the division apparatus (e.g., FtsZ) to compensate for the lack of the candidate. These experimental approaches do not yet exist for Chlamydia. Thus, to further explore the function of Ct009 as a RodZ homolog, we used a complementing approach in a surrogate E. coli RodZ mutant strain (FB60/pTB63) where rodZ has been deleted, but 
A

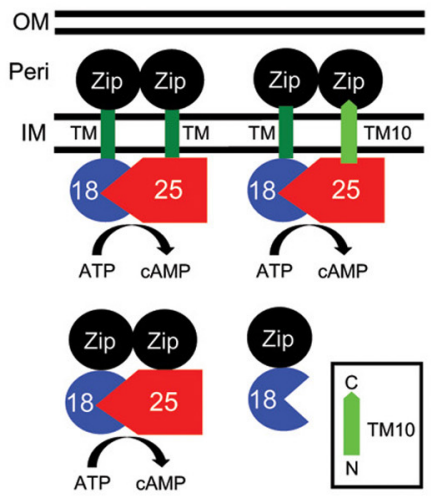

B

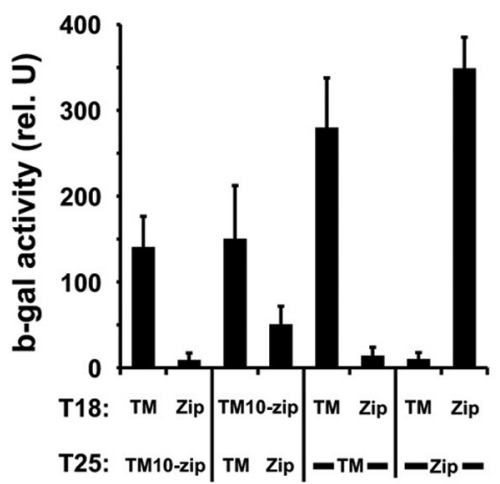

C

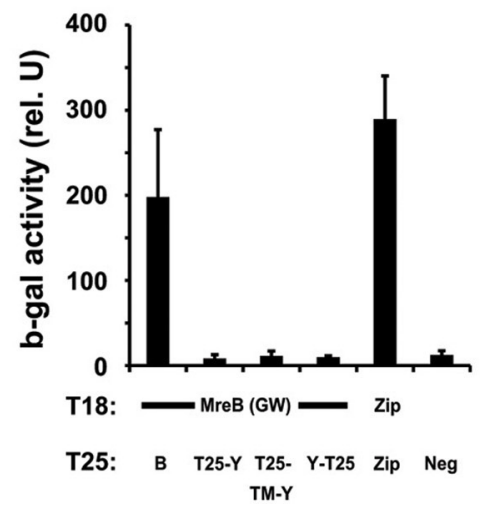

FIGURE 2 | Determination of the orientation of MraY and its interaction with MreB using the BACTH system. (A) The 26 amino acids encoding TM10 of MraY (residues 311-336; light green with predicted orientation boxed in bottom right of $\mathbf{A}$ ) were cloned between the leucine zipper domain, Zip (black circle), and the T18 (blue pie shape) or T25 (red pentagon) fragment of adenylate cyclase to create the product T25-TM10-zip or T18-TM10-zip. These were tested against the positive control vectors encoding T25-Zip or T18-Zip and T25-TM-zip or T18-TM-zip (indicated as TM in $(\mathbf{B}, \mathbf{C})$ with the transmembrane domain of OppB from E. coli; dark green). The diagram illustrates the expected interactions with T18-TM-zip if T25-TM10-zip has its C-terminus in the periplasm as predicted. Note that the Zip domains must bring together the T18 and T25 fragments to detect an interaction. IM, inner membrane; Peri, Periplasm; OM, outer membrane. (B) Quantification of the ß-galactosidase activity from the BACTH tests of TM10 from MraY as described in (A). (C) Chlamydial MreB does not interact with MraY. T18-MreB(GW) was tested against T25-MreB(GW) [B] or T25-MraY [T25-Y], T25-TM-MraY expressed from the BACTH-TM plasmid [T25-TM-Y], or MraY-T25 [Y-T25]. Note that only the T25-TM-MraY fusion respects the native topology of MraY as an extra TM is inserted between the cytosolic T25 domain and the N-terminal extremity of MraY. ß-galactosidase assays were performed as described in the legend to Figure $\mathbf{1}$. the cells are kept viable by the presence of a plasmid encoding ftsQAZ (Bendezú et al., 2009). In this RodZ- background, the bacteria lose their rod-shape morphology and are round or irregularly shaped but continue to divide. GFP-Ct009 or GFPRodZ (from E. coli) was expressed in either a RodZ wild-type background or the $\Delta \operatorname{rod} Z$ mutant strain. In wild-type cells, GFPCt009 localized uniformly to the membranes whereas GFP-RodZ from E. coli adopted the characteristic spotty helical-like membrane localization pattern consistent with previous observations (Figure 5; Bendezú et al., 2009). In the $\Delta \operatorname{rodZ}$ cells, GFP-Ct009 showed the same membrane localization pattern as in wildtype cells, and it failed to correct their shape defects (Figure 5). Conversely, GFP-RodZ from E. coli was able to do so and again showed its characteristic localization pattern. The empty vector control showed no effect on the $\Delta \operatorname{rod} Z$ mutant strain, as expected (Supplemental Figure S2). Thus, Ct009 correctly localizes to the membrane in E. coli but is unable to complement the shape defect of $E$. coli cells that lack native RodZ.

\section{FAILURE OF Ct009/RodZ TO COMPLEMENT MAY BE DUE TO ITS INABILITY TO RECAPITULATE ESSENTIAL INTERACTIONS}

To explore in further detail why chlamydial RodZ failed to complement the E. coli depletion mutant, we tested heterologous interactions between the chlamydial and E. coli RodZ and MreB proteins. Pair-wise BACTH interaction tests were performed between these proteins in each orientation and combination (Figure 6). Chlamydial MreB interacted robustly with chlamydial RodZ in all combinations. Chlamydial MreB also interacted efficiently with E. coli MreB, perhaps not surprising given the high level of conservation (57\% identical/71\% similar over 95\% of E. coli MreB; E value = 1e-125). Further, chlamydial MreB interacted with E. coli RodZ but only when the latter was encoded on the low copy vector (i.e., with T25-RodZ). Conversely, chlamydial RodZ failed to show an interaction with E. coli MreB or RodZ and was not capable of dimerizing as was shown for other RodZ homologs (Figure 6, Supplemental Figure S3; White et al., 2010). We conclude that the lack of complementation of the E. coli $\triangle \operatorname{rod} Z$ mutant by $\mathrm{Ct} 009$ is most likely due to its inability to recapitulate essential interactions with the $E$. coli shape determining protein MreB.

\section{DISCUSSION}

Chlamydia is an important human pathogen that presents certain challenges with which to work. A complicating factor for performing interaction or localization studies is the relatively small size of the organism and its obligate dependence on a host cell for growth. Characterization of interacting properties of chlamydial proteins is one approach to gain further insight about the unique biology of this pathogen. Therefore, we used a bacterial two-hybrid system, the $\mathrm{BACTH}_{\mathrm{GW}}$, to examine chlamydial protein-protein interactions, with a particular emphasis on the study of cell division proteins. Intriguingly, Chlamydia is remarkable in lacking the highly conserved and essential FtsZ protein that plays a key role in organizing the bacterial divisome (Begg and Donachie, 1985). We proposed recently that the cytosolic protein MreB may serve a similar role as a functional substitute for FtsZ in chlamydial cell division (Ouellette et al., 2012). Here, we searched for additional chlamydial partners of MreB, hypothesizing that they might be putative cell division proteins in this bacterium. We found that Ct009, a membrane protein with homology to RodZ/YfgA, efficiently interacted with chlamydial MreB. We are currently pursuing further $\mathrm{BACTH}_{\mathrm{GW}}$ screens to 


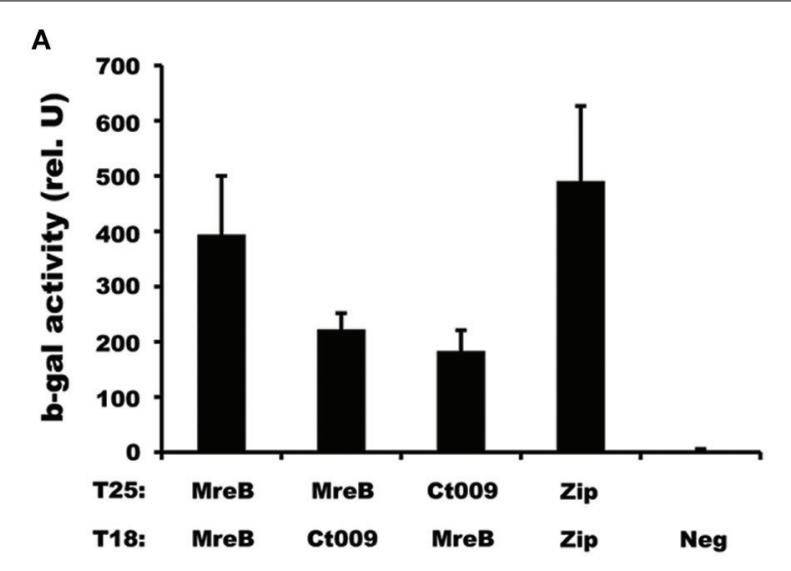

C

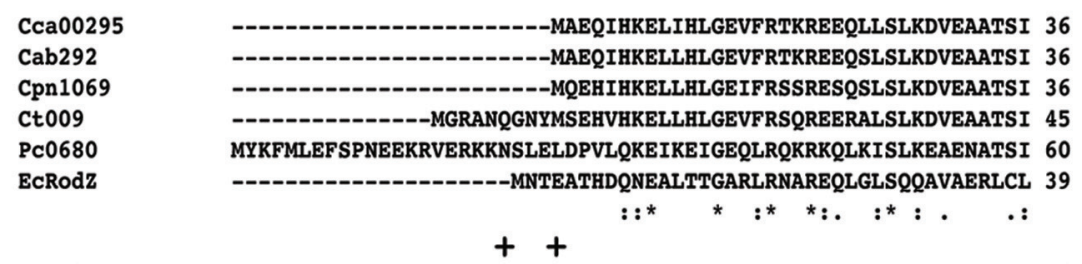

Cca00295

Cab292

Cpn1069

Ct009

Pc0680

EcRodz

Cca00295

Cab292

Cpn1069

Ct009

Pc0680

EcRodz RYSCLEAIENGYLGKLISPIYAOFIKKY RYSCLEAIEQGCLGKLISPVYAQGF IKKYATYLGLDGDSILQEHPYVMKIFKEFSDHNME 96 RLSALEAIEAGHLGKLISPVYAQGFMKKYAAFLDMDGDRLLKEHPYVLKIFQEFSDQNMD 105 RMVYLQALEEGEMEKLISPVYAQGFFKQYASFLGIDGESIIREKPYIFN-----RPEAQE 115 KVSTVRDIEEDKAPADLASTFLRGYIRSYARLVHIPEEELLPG-------LEKQAPLRAA 92

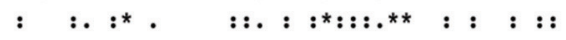

MLLDLESMGGRNSPEKAIRTWSNLWWVALITISGMA-IWWLGSLLSIF------------- 143 MLLDLESMGGRNSPEKAIRSLSNLWWAGLIVFSGMA-IWWLGSLLSLF------------- 143 MLLDLESMGGRNSPERAIHSWSNLWWAGLIIIGGIM-VWWLGSLFSIF------------- 143 MLLDLESMGGRNSPEKAIRSWLNLGWAGVFVVGAAC-IWWLGNLFNLF------------ 152 FAYGIGTLEVRSNPGASVKWVPNVMWVLAFMGVLFA-AWYVAKILGVL------------- 162 KVAPMQSFSLGKRRKKRDGWLMTFTWLVLFVVIGLSGAWWWQDRKAQQEEITTMADQSSA 152
B

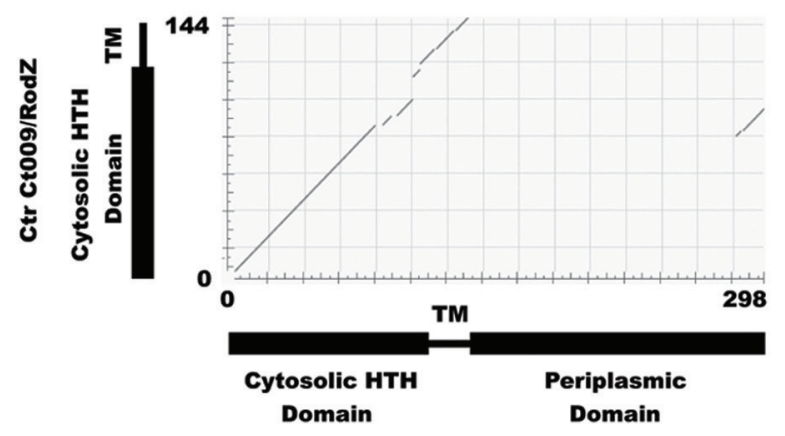

E. coli Rodz

D

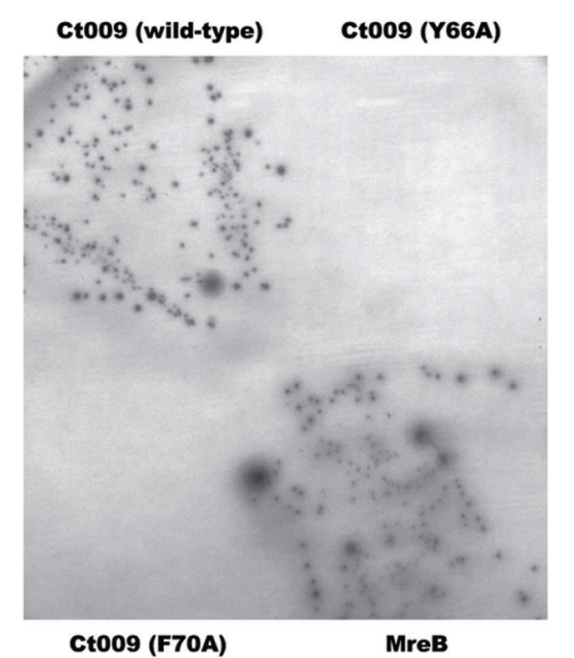
: : : . ..* : ${ }^{*}$ * .

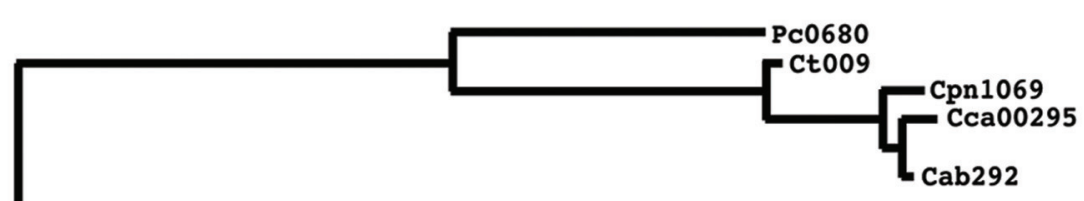

ECRodz

FIGURE 3 | Identification of a chlamydial rodZ homolog. (A) Interaction of chlamydial MreB with $\mathrm{Ct009}$ in the $\mathrm{BACTH}_{\mathrm{GW}}$ system. A positive control of T25-Zip vs. T18-Zip is shown. The ß-galactosidase assays and negative controls are as described for Figure 1. (B) Alignment of chlamydial Ct009/RodZ (y-axis) with E. coli RodZ (x-axis) with corresponding domains schematically represented. Note that the chlamydial RodZ homolog is missing the periplasmic domain. (C) ClustalW sequence alignment and phylogenetic relatedness of chlamydial Ct009 homologs, with corresponding gene numbering, vs. amino acids 1-152 of E. coli RodZ with conserved residues indicated by $\left[{ }^{*}\right]$ and related residues by dots (e.g., [:]). The "+" indicates the MreB-interaction residues as described by van den Ent et al. (2010). Cca, C. caviae; Cab, C. abortus; Cpn, C. pneumoniae; Ct, C. trachomatis; Pc, Protochlamydia ameobophila; Ec, E. coli (D) BACTH test of T25-MreB vs. the indicated T18 fusion proteins. Only positive interactions lead to growth on minimal medium with maltose with corresponding production of ß-galactosidase. identify additional components of the chlamydial cell division apparatus.

The identification of a RodZ homolog in Chlamydia adds to the repertoire of rod-shape determining proteins in this coccoid bacterium. Chlamydia has undergone extensive genome reduction in adapting to its obligate intracellular life style. Every open reading frame is transcriptionally expressed during the developmental cycle (Belland et al., 2003), and there are few pseudogenes present in chlamydial genomes (Stephens et al., 1998). Therefore, the majority of genes are likely essential. Consequently, the presence of rod-shape determining genes in Chlamydia should be interpreted as necessary for its survival. In bacilli, the rodshape determining proteins direct the peptidoglycan machinery to the cell wall to allow for side-wall growth whereas a different 

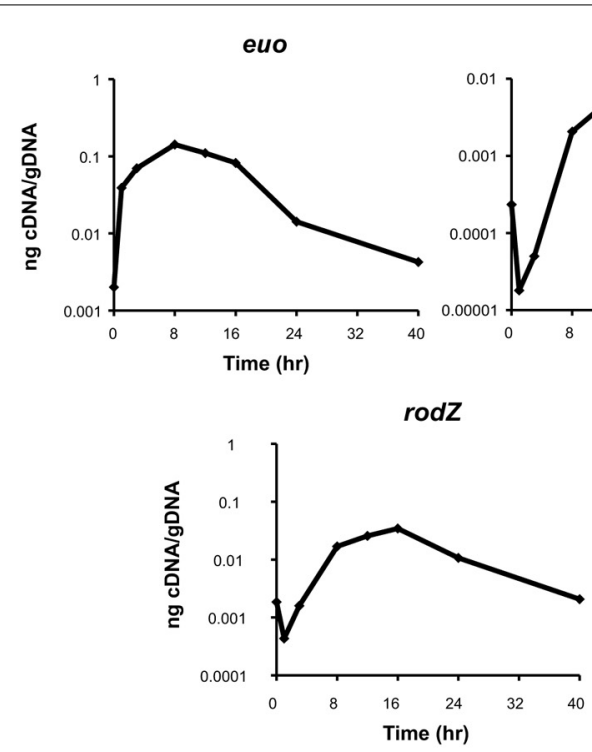

FIGURE 4 | Transcriptional analysis of ct009/rodZ during the chlamydial developmental cycle. The indicated genes were analyzed by RT-qPCR over a time course of infection with $C$. trachomatis. The top panels show control genes for EB-to-RB transition (euo), RB growth and division (ftsl), and RB-to-EB transition (omcB). Transcription of $\operatorname{rod} Z$ and $m r e B$ is shown in the
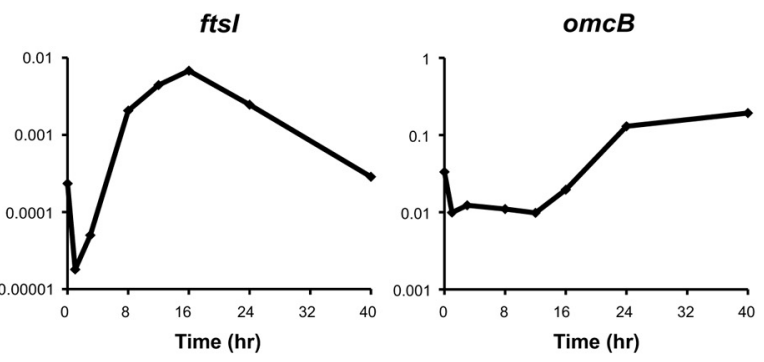

mreB

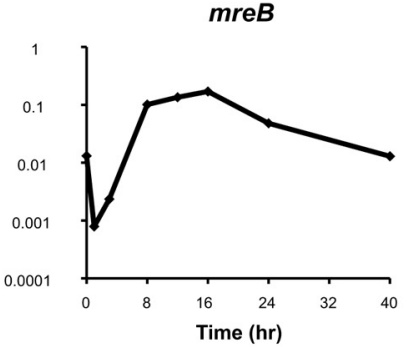

bottom panels and exhibits an fts/-like expression pattern indicative of RB growth and division. DNase-treated total RNA was reverse transcribed and analyzed by qPCR. Data were normalized to genome numbers and are representative of two biological replicates analyzed in triplicate. Standard deviations were generally less than $5 \%$ of the average.

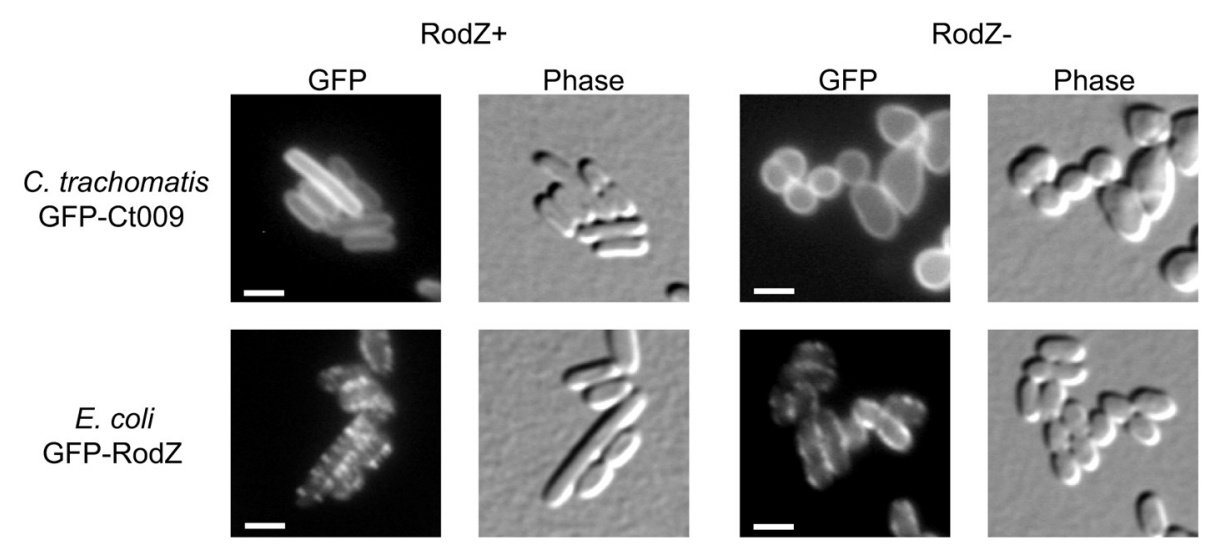

FIGURE 5 | Test of complementation of Ct009/RodZ in E. coli. An E. coli wild-type strain (RodZ+) or the $\Delta$ rodZ FB60/pTB63 mutant E. coli strain (RodZ-) was transformed with the indicated GFP constructs. Expression was induced and the localization of the RodZ protein and the cell shape were monitored by fluorescence microscopy. The absence of RodZ leads to loss of the rod shape with resulting irregular coccoid morphology. Scale bar $=2 \mu \mathrm{m}$. set of machinery is used to generate a cell wall (i.e., septum) in the division plane (van der Ploeg et al., 2013). Chlamydia lacks many of the canonical cell division proteins including FtsZ, thus we have hypothesized that it has co-opted the rodshape determining proteins to function in cell division (Ouellette et al., 2012). Interestingly, although Chlamydia encodes a RodZ homolog, Ct009, this protein was unable to complement an E. coli RodZ mutant in spite of its localization to membranes. Given the inability of chlamydial $\operatorname{Rod} Z$ to re-capitulate the essential interaction with E. coli MreB, this finding is perhaps not surprising. Further, given the coccoid shape of Chlamydia, it stands to reason that the chlamydial $\operatorname{RodZ}$ may have lost essential determinants that govern the rod-shape and/or gained other determinants that support a different function in Chlamydia. We are currently exploring this.

Very recently, Jacquier et al. (2014) have shown that a Waddlia homolog of Ct009 localizes to apparent division sites in this bacterium. Given our interaction data showing that MreB interacts with Ct009/RodZ, these data combine to show a likely role of chlamydial RodZ in cell division. Based on combinatorial antibiotic treatments as we have previously used (Ouellette et al., 2012), Jacquier et al. (2014) also suggested that MreB functions downstream from peptidoglycan precursors in the division process. Part of their rationale for this conclusion is based on 


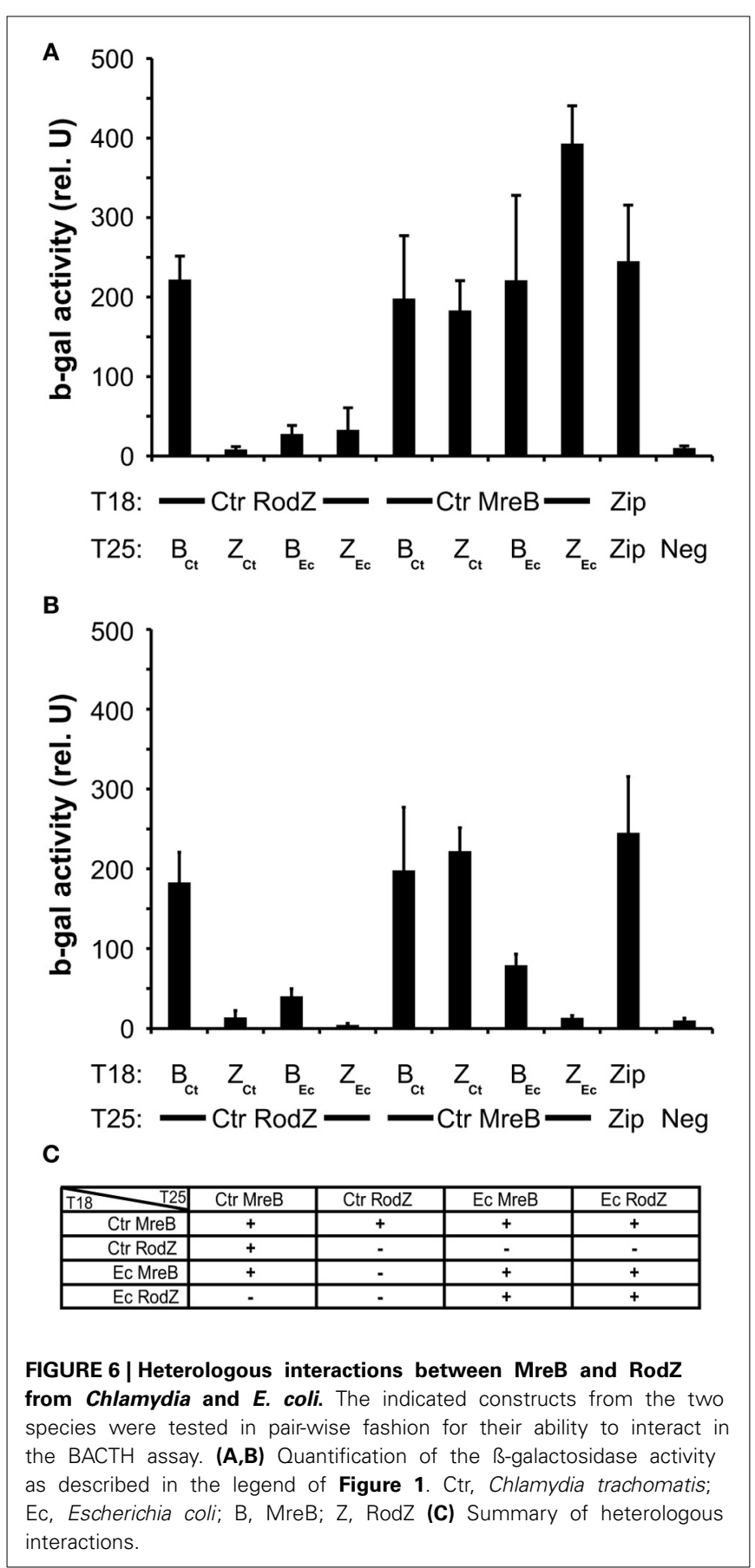

the supposed interaction of MreB with MraY and MurG, both of which are involved in peptidoglycan synthesis, as reported by Gaballah et al. (2011). These authors also used the BACTH assay to suggest that MraY and MurG could bind to MreB in Chlamydia pneumoniae. However, the BACTH interaction assays of Gaballah et al. (2011) yielded only very low levels of $\beta$ galactosidase activity (less than twice the negative control levels whereas a positive interaction should have 5 -fold greater activity). Thus, the interpretation of MreB's interacting with MraY and MurG from these data is flawed. One major caveat with the MraY study is that $\mathrm{MraY}$ is predicted to have both its $\mathrm{N}$ - and C-termini in the periplasm (Bouhss et al., 1999; Chung et al., 2013). Thus, direct fusion of the T25 or T18 cyclase fragments to MraY, as done by Gaballah et al. (2011), is expected to result in a non-native insertion of the protein into the membrane, or alternatively, in the targeting of the T25 or T18 fragment to the periplasm where they would not be functional. We did not detect interactions between MreB and MraY or MurG using the same experimental approach as Gaballah et al. (2011) and argue that the interpretation of their data is incorrect. Consequently, any conclusions based on their data should be carefully interpreted, and further experimental work will be required to establish such an interaction.

In sum, we have shown the utility of examining proteinprotein interactions to identify components of pathways in Chlamydia that were previously not annotated. Without being able to implement conditional knockout or depletion systems, this approach will aid in our understanding of chlamydial microbiology. Indeed, for proteins that are essential in Chlamydia, this may be the preferred approach.

\section{AUTHOR CONTRIBUTIONS}

Scot P. Ouellette designed and performed experiments, analyzed data, and wrote the manuscript. Kelsey J. Rueden, Emilie Gauliard, and Logan Persons performed experiments. Piet A. de Boer and Daniel Ladant designed experiments, analyzed data, and wrote the manuscript.

\section{ACKNOWLEDGMENTS}

These data were presented, in part, at the EMBO Workshop, "Reconstructing the essential bacterial cell cycle machinery," in Real Sitio de San Ildefonso, Spain from the 16th to the 19th of September, 2012. The authors would like to thank the Pathogen Functional Genomic Resource Center (PFGRC) for providing glycerol stocks of the library of Chlamydia genes cloned into pDONR221. Scot P. Ouellette was partially supported by a fellowship from the Pasteur Foundation and start-up funds from the University of South Dakota. Emilie Gauliard was supported by a fellowship from the Université Paris Diderot. Piet A. de Boer was supported by NIH grant GM57059.

\section{SUPPLEMENTARY MATERIAL}

The Supplementary Material for this article can be found online at: http://www.frontiersin.org/journal/10.3389/fmicb.2014. 00279/abstract

Supplemental Figure S1 | TOPCONS membrane topological analysis of chlamydial MraY (Ct757). Note the presence of 10 TM domains with both the $\mathrm{N}$ - and C-termini located "outside."

Supplemental Figure S2 | The empty vector pMLB1113 $\Delta$ H3 has no effect on the cell shape of the $\Delta$ rodZ mutant strain, FB60/pTB63. Cells from the transformed strain were imaged under phase contrast.

Supplemental Figure S3 | BACTH interactions between E. coli MreB and RodZ. Quantification of the ß-galactosidase activity as described in the legend of Figure 1

Supplemental Table S1 | List of primers, plasmids, and strains used in the study. 


\section{REFERENCES}

AbdelRahman, Y. M., and Belland, R. J. (2005). The chlamydial developmental cycle. FEMS Microbiol. Rev. 29, 949-959. doi: 10.1016/j.femsre.2005. 03.002

Alyahya, S. A., Alexander, R., Costa, T., Henriques, A. O., Emonet, T., and Jacobs-Wagner, C. (2009). RodZ, a component of the bacterial core morphogenic apparatus. Proc. Natl. Acad. Sci. U.S.A. 106, 1239-1244. doi: 10.1073/pnas.0810794106

Battesti, A., and Bouveret, E. (2012). The bacterial two-hybrid system based on adenylate cyclase reconstitution in Escherichia coli. Methods 58, 325-334. doi: 10.1016/j.ymeth.2012.07.018

Begg, K. J., and Donachie, W. D. (1985). Cell shape and division in Escherichia coli: experiments with shape and division mutants. J. Bacteriol. 163, 615-622.

Belland, R. J., Zhong, G., Crane, D. D., Hogan, D., Sturdevant, D., Sharma, J., et al. (2003). Genomic transcriptional profiling of the developmental cycle of Chlamydia trachomatis. Proc. Natl. Acad. Sci. U.S.A. 100, 8478-8483. doi: 10.1073/pnas. 1331135100

Bendezú, F. O., Hale, C. A., Bernhardt, T. G., and de Boer, P. A. (2009). RodZ (YfgA) is required for proper assembly of the MreB actin cytoskeleton and cell shape in E. coli. EMBO J. 28, 193-204. doi: 10.1038/emboj.2008.264

Bernsel, A., Viklund, H., Hennerdal, A., and Elofsson, A. (2009). TOPCONS: consensus prediction of membrane protein topology. Nucleic Acid Res. 37, W465-W468. doi: 10.1093/nar/gkp363

Bork, P., Sander, C., and Valencia, A. (1992). An ATPase domain common to prokaryotic cell cycle proteins, sugar kinases, actin, and hspt70 heat shock proteins. Proc. Natl. Acad. Sci. U.S.A. 89, 7290-7294. doi: 10.1073/pnas.89.16.7290

Bouhss, A., Mengin-Lecreulx, D., Le Beller, D., and Van Heijenoort, J. (1999). Topological analysis of the MraY protein catalysing the first membrane step of peptidoglycan synthesis. Mol. Microbiol. 34, 576-585. doi: 10.1046/j.13652958.1999.01623.x

Chung, B. C., Zhao, J., Gillespie, R. A., Kwon, D.-Y., Guan, Z., Hong, J., et al. (2013). Crystal structure of MraY, an essential membrane enzyme for bacterial cell wall synthesis. Science 341, 1012-1016. doi: 10.1126/science.1236501

Doi, M., Wachi, M., Ishino, F., Tomioka, S., Ito, M., Sakagami, Y., et al. (1988). Determinations of the DNA sequence of the $m r e B$ gene and of the gene products of the mre region that function in formation of the rod shape of Escherichia coli cells. J. Bacteriol. 170, 4619-4624.

Everett, K. D., and Hatch, T. P. (1991). Sequence analysis and lipid modification of the cysteine-rich envelope proteins of Chlamydia psittaci 6BC. J. Bacteriol. 173, 3821-3830.

Gaballah, A., Kloeckner, A., Otten, C., Sahl, H. G., and Henrichfreise, B. (2011). Functional analysis of the cytoskeleton protein MreB from Chlamydophila pneumoniae. PLoS ONE 6:e25129. doi: 10.1371/journal.pone.0025129

Jacquier, N., Frandi, A., Pillonel, T., Viollier, P., and Greub, G. (2014). Cell wall precursors are required to organize the chlamydial division septum. Nat. Commun. 5:3578. doi: $10.1038 /$ ncomms 4578

Johnson, J. E., Lackner, L. L., and de Boer, P. A. J. (2002). Targeting of MinC/MinD and MinC/DicB complexes to septal rings in Escherichia coli suggests a multistep mechanism for MinC-mediated destruction of nascent FtsZ-rings. J. Bacteriol. 184, 2951-2962. doi: 10.1128/JB.184.11.2951-2962.2002

Karimova, G., Dautin, N., and Ladant, D. (2005). Interaction network among Escherichia coli membrane proteins involved in cell division as revealed by bacterial two-hybrid analysis. J. Bacteriol. 187, 2233-2243. doi: 10.1128/JB.187.7.2233-2243.2005

Karimova, G., Pidoux, J., Ullmann, A., and Ladant, D. (1998). A bacterial twohybrid system based on a reconstituted signal transduction pathway. Proc. Natl. Acad. Sci. U.S.A. 95, 5752-5756. doi: 10.1073/pnas.95.10.5752

Mabey, D. C. W., Solomon A. W., and Foster A. (2003). Trachoma. Lancet 362, 223-229. doi: 10.1016/S0140-6736(03)13914-1

Mukherjee, A., Dai, K., and Lutkenhaus, J. (1993). Escherichia coli cell division protein FtsZ is a guanine nucleotide binding protein. Proc. Natl. Acad. Sci. U.S.A. 90, 1053-1057. doi: 10.1073/pnas.90.3.1053
Ouellette, S. P., AbdelRahman, Y. M., Belland, R. J., and Byrne, G. I. (2005). The Chlamydia pneumoniae type III secretion-related $l c r H$ gene clusters are developmentally expressed operons. J. Bacteriol. 187, 7853-7856. doi: 10.1128/JB.187.22.7853-7856.2005

Ouellette, S. P., Gauliard, E., Antosova, Z., and Ladant, D. (2014). A Gateway ${ }^{\circledR}$ compatible bacterial adenylate cyclase-based two hybrid system. Environ. Microbiol. Rep. 6, 259-267. doi: 10.1111/1758-2229.12123

Ouellette, S. P., Hatch, T. P., AbdelRahman, Y. M., Rose, L. A., Belland, R. J., and Byrne, G. I. (2006). Global transcriptional upregulation in the absence of increased translation in Chlamydia during IFNgamma-mediated host cell tryptophan starvation. Mol. Microbiol. 62, 1387-1401. doi: 10.1111/j.13652958.2006.05465.x

Ouellette, S. P., Karimova, G., Subtil, A., and Ladant, D. (2012). Chlamydia coopts the rod-shape determining proteins MreB and $\mathrm{Pbp} 2$ for cell division. Mol. Microbiol. 85, 164-178. doi: 10.1111/j.1365-2958.2012.08100.x

Schachter, J., Storz, J., Tarizzo, M. L., and Bogel, K. (1973). Chlamydiae as agents of human and animal diseases. Bull. World Health Organ. 49, 443-449.

Seppala, S., Slusky, J. S., Lloris-Garcera, P., Rapp, M., and von Heijne, G. (2010). Control of membrane protein topology by a single C-terminal residue. Science 328, 1698-1700. doi: 10.1126/science. 1188950

Shaw, E. I., Dooley, C. A., Fischer, E. R., Scidmore, M. A., Fields, K. A., and Hackstadt, T. (2000). Three temporal classes of gene expression during the Chlamydia trachomatis developmental cycle. Mol. Microbiol. 37, 913-925. doi: 10.1046/j.1365-2958.2000.02057.x

Shiomi, D., Sakai, M., and Niki, H. (2008). Determination of bacterial rod shape by a novel cytoskeletal membrane protein. EMBO J. 27, 3081-3091. doi: 10.1038/emboj.2008.234

Stephens, R. S., Kalman, S., Lammel, C., Fan, J., Marathe, R., Aravind, L., et al. (1998). Genome sequence of an obligate intracellular pathogen of humans: Chlamydia trachomatis. Science 282, 754-759. doi: 10.1126/science.282.5389.754

van den Ent, F., Amos, L. A., and Lowe, J. (2001). Prokaryotic origin of the actin cytoskeleton. Nature 413, 39-44. doi: 10.1038/35092500

van den Ent, F., Johnson, C. M., Persons, L., de Boer, P., and Lowe, J. (2010). Bacterial actin MreB assembles in complex with cell shape protein RodZ. EMBO J. 29, 1081-1090. doi: 10.1038/emboj.2010.9

van der Ploeg, R., Verheul, J., Vischer, N. O., Alexeeva, S., Hoogendoorn, E., Postma, M., et al. (2013). Colocalization and interaction between elongasome and divisome during a preparative cell division phase in Escherichia coli. Mol. Microbiol. 87, 1074-1087. doi: $10.1111 / \mathrm{mmi} .12150$

White, C. L., Kitich, A., and Gober, J. W. (2010). Positioning cell wall synthetic complexes by the bacterial morphogenetic proteins MreB and MreD. Mol. Microbiol. 76, 616-633. doi: 10.1111/j.1365-2958.2010.07108.x

Wichlan, D. G., and Hatch, T. P. (1993). Identification of an early-stage gene of Chlamydia psittaci 6BC. J. Bacteriol. 175, 2936-2942.

Conflict of Interest Statement: The authors declare that the research was conducted in the absence of any commercial or financial relationships that could be construed as a potential conflict of interest.

Received: 21 February 2014; accepted: 21 May 2014; published online: 06 June 2014. Citation: Ouellette SP, Rueden KJ, Gauliard E, Persons L, de Boer PA and Ladant $D$ (2014) Analysis of MreB interactors in Chlamydia reveals a RodZ homolog but fails to detect an interaction with MraY. Front. Microbiol. 5:279. doi: 10.3389/fmicb. 2014.00279

This article was submitted to Microbial Physiology and Metabolism, a section of the journal Frontiers in Microbiology.

Copyright (c) 2014 Ouellette, Rueden, Gauliard, Persons, de Boer and Ladant. This is an open-access article distributed under the terms of the Creative Commons Attribution License (CC BY). The use, distribution or reproduction in other forums is permitted, provided the original author(s) or licensor are credited and that the original publication in this journal is cited, in accordance with accepted academic practice. No use, distribution or reproduction is permitted which does not comply with these terms. 